\title{
Long-term care provision in Europe: Results of a desk research study
}

\author{
Slavena Manolova ${ }^{1}$ @ hhtps://rcid.orgy $0000-0002-9917-9703$ \\ Milena Pavlova ${ }^{1}$ (1) https://orid.orgy000-0002-6082-8446

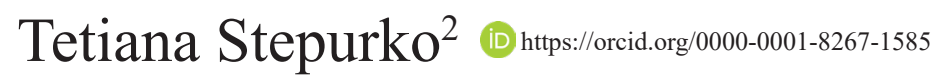 \\ Marzena Tambor ${ }^{3}$ (I htpps:/orcid.org 0000-0001--7970-6919 \\ Wim Groot ${ }^{1}$ (ํ) htpp:/orcid.orgy0000-0003-1035-5916
}

${ }^{1}$ Department of Health Services Research, CAPHRI, Faculty of Health, Medicine and Life Sciences, Maastricht University, Maastricht, the Netherlands

2 School of Public Health, National University of Kyiv-Mohyla Academy, Kyiv, Ukraine

${ }^{3}$ Department of Health Economics and Social Security, Institute of Public Health, Faculty of Health Sciences, Jagiellonian University Collegium Medicum, Cracow, Poland

Address for correspondence: Milena Pavlova, Department of Health Services Research, Faculty of Health, Medicine and Life Sciences, Maastricht University, PO Box 616, 6200 MD Maastricht, the Netherlands, +31 433881705 , m.pavlova@maastrichtuniversity.nl

\section{Abstract}

This paper describes the provision of long-term care across Europe based on data gathered in a desk research. The aim is: (1) to identify indicators of long-term care provision; and (2) to compare the provision of formal and informal care across the European countries. For this purpose, a narrative literature review was carried out to identify relevant indicators. Subsequently, a descriptive analysis was performed to analyse the indicatorrelated data. The results suggested that there are important differences in the long-term care provision in Europe. Long-term care is provided both at public and private institutions. The entitlement criteria vary among countries. In general, Western and Northern European countries have more generous provision of residential care compared to Eastern and Southern European countries. At the same time, informal care has different roles and it is extremely important in Eastern and Southern European countries. Among all countries, more than half have quality assurance regulations for residential care. However, most of the Southern and Eastern European countries lack information about the quality assurance regulations. In order to monitor the long-term care provision, it is recommended that European countries establish a reporting system to provide annual data. These annual data should be based on identical measurement mechanisms and standardised reporting structure to allow for comparison and improvements of long-term care systems.

\section{Key words: desk research, Europe, funding, long-term care, management, provision}

Slowa kluczowe: analiza danych zastanych, Europa, finansowanie, opieka dtuyoterminowa, zarzadzanie, dostarczanie

\section{Acknowledgements}

Milena Pavlova is the recipient of an AXA Award of the AXA Research Fund, Project "EuroLTCS Project - Innovative Mechanisms for Sustainable Long-Term Care Systems in Europe". The content of this publication represents the views of the authors and it is their sole responsibility; it can in no way be taken to reflect the views of the AXA Research Fund. 


\section{Introduction}

European countries are facing significant challenges within their health care and social care systems caused by demographic changes. In particular, the ageing of the population questions the capacity of these systems to respond to the specific care needs. The increased demand for care requires more care providers and more financial resources [1]. Therefore, effective policy actions are needed to contain the costs and to assure an adequate care supply, as well as to sustain the long-term care (LTC) systems. The challenges posed by the demographic changes have been acknowledged and debated upon by the EU and relevant stakeholders. Moreover, the consequences of the population ageing, such as increased LTC needs, stay high on the EU's agenda [2].

LTC is a broad term and its definitions vary. LTC is defined by the WHO as the group of activities performed by informal caregivers and/or formal professionals to ensure that an individual incapable of coping and sustaining self-care "can maintain the highest possible quality of life, according to his or her individual preferences, with the greatest possible degree of independence, autonomy, participation, personal fulfilment and human dignity" [3]. Another common definition of LTC is the one by Colombo et al. [4], who defined LTC as a range of services required by persons with a reduced degree of functional capacity, physical or cognitive, and who are dependent for an extended period of time on help with basic activities of daily living (ADL) such as eating, bathing, washing, dressing, getting in and out of bed, getting to and from the toilet as well as so-called instrumental activities of daily living (IADL) (e.g., shopping, laundry, cooking, performing housework etc.). These services are frequently provided in combination with basic medical services - nursing care (e.g. wound dressing, pain management, medication, health monitoring), as well as health protection, rehabilitation or palliative care. Hence, LTC is provided within two sectors - health care and social care sector. In health care, medical services for LTC users are provided, while the social sector is responsible for personal LTC services (help with ADL) and social services (help with IADL). An important aspect of LTC is that care is typically provided for an extended period of time [5]. The duration and level of LTC depends on the individual's condition and could change over time. The older population groups aged 80 and above, are the most frequent users of LTC [6], but LTC could also be provided for younger people (under 65 years) with physical or mental disabilities.

Provision of LTC might be of formal or informal nature. Formal care is related to diverse systems of public and private services (social care services, health care services, rehabilitation services, and palliative care) which are provided in the context of formal employment $[3,6]$. Formal LTC may be delivered at different living settings including home of the recipient (home care), institutional facilities, e.g. nursing homes, hospices and other residential care facilities, or day-care facilities $[5,7]$. Formal caregivers are usually professional assistants or nurses who provide support at an institution or at the house of the LTC recipient. On the other hand, informal care is usually provided at home by unpaid non-professional caregivers, who may be spouses, other family members, friends or neighbours of the recipient.

There are differences in the coverage and provision of LTC across EU Member States [1]. Comparative analyses of the LTC provision mechanisms across countries could help to identify the advantages and disadvantages, and suggest key areas for improvement. However, due to the complex nature and diversity of LTC systems, it is difficult to make international comparisons of the care provision [1].

Although a few studies related to the typology of financing and provision of LTC systems in the EU, have been performed, they are limited to only a couple of European countries [8,9]. In this study, the aim is: (1) to identify indicators of long-term care provision; and (2) to compare the provision of formal and informal care across the European countries. We include the EU Member States as well as countries, which are members of the European Economic Association (EEA); Iceland, Norway and Switzerland. For this purpose, a range of indicators is identified in a narrative literature review and is used to measure the provision of LTC in the EU/EEA area.

\section{Methods}

The data for this study were collected in a desk research conducted in May-June 2017. In particular, a narrative literature review of EU, OECD and WHO databases and publications was conducted to identify and describe the available indicators of LTC provision. Scientific and policy reports were also reviewed to find additional data. Overall, the study had an explorative and descriptive nature.

In particular, the international databases of the EU, OECD and WHO, which covered the health statistics, were searched to identify relevant indicators of LTC provision and the values of those indicators for European countries. However, a significant drawback of these data sources was that they did not include all countries in the EU/EEA area. They also covered different years, and the most recent data were sometimes absent. Therefore, cross-country reports found on the websites of OECD, WHO and EU were also used to crosscheck and complete the information on LTC provision indicators. The recently published Joint Report on Health Care and LTC Systems [1] by the European Commission was one of the primary sources of data for our review. Other relevant sources were also identified during the data collection $[4$, 10-13].

In addition, we reviewed relevant scientific and policy reports to complete missing information on LTC provision indicators. These reports were identified in a search in PubMed. The following search terms were included: long-term care in Europe, provision of long-term care, quality of long-term care, access to long-term care, formal and informal providers of LTC, European LTC systems. In addition, the following inclusion and exclusion criteria were defined: only English language publications 
that appeared after 2008 and could be downloaded (full text available, not just abstract), were considered for inclusion. Regarding the type of publications, research article, policy report and book chapter were considered. After the review of the full text, a publication was included in the review if it provided data on LTC provision indicator(s) of EU/EEA countries.

The triangulation of the different sources of data, namely the EU, OECD and WHO databases and publications, as well as other scientific and policy reports, helped us to improve the reliability and validity of the study. Since the information for the study needed to be as relevant as possible, only the latest available data were used in the analysis. To assure the consistency of the estimations, an indicator was only used if we were able to find values for that indicator for most of the EU/EEA countries.

The LTC provision indicators identified in our narrative review were divided into indicators of formal and informal care provision as suggested in previous literature $[1,14,15]$. The results of the data analysis are presented in the form of a narrative description illustrated with tables.

\section{Results}

Our review resulted in several indicators of formal and informal LTC provision in the EU and EEA countries. We divided these indicators into four sub-groups: (1) general characteristics of LTC provision; (2) provision of formal LTC; (3) provision of informal LTC; (4) regulation/policy related to LTC quality assurance, health protection and rehabilitation. We subsequently describe the results per sub-group.

Table I presents indicators related to the general characteristics of LTC provision that we found in the review:

- Entitlement to LTC (population groups to whom the right to LTC benefits is granted).

- Age criterion for LTC use.

- Qualifying period for LTC use (minimum period of residence in the country or insurance membership before a person receives the right to use LTC).

- Public expenditure on LTC as \% of GDP.

- Type of care providers (public or private providers).

- Total number of formal LTC nurses and personal carers.

- Number of LTC beds in hospitals.

- Number of LTC beds in nursing and other residential care facilities.

- Number of LTC beds in nursing and other residential care facilities per hundred thousand inhabitants.

Regarding the entitlement to LTC, the results in Table I show that only a few countries provide access to all residents without specific criteria. The rest of the countries have established specific criteria for LTC, such as the ADL and IADL, disability and severe disability levels, or residents under insurance.

In all countries, the entitlement to LTC is dependent on the services or benefit, which are needed. The need of LTC is however defined and established very differ- ently across the countries, ranging from the application of strict criteria defined in advance to the assessment of the individual situation of the person requesting LTC. In addition, in two thirds of the countries, there are no age conditions or restrictions for the use of LTC. Only Romania has identified the standard retirement age as a condition for LTC, Additionally, Malta has a condition of being at least 60 years old to use LTC, while in Estonia, the UK and Hungary, LTC is possible from the age of 18. Regarding the qualifying period required for LTC use, the majority of countries do not apply this criterion. Germany, Ireland, the UK and Iceland have a qualifying period for LTC use, while in Austria, Belgium, Luxembourg, the Netherlands, Spain and Switzerland, such period is only applied depending on the type of insurance, residence, or the reason for LTC.

The data indicate that among all countries, the Netherlands is spending most on LTC as a percentage of GDP. In addition, Sweden, Norway, Switzerland, Denmark and Belgium also have high public expenditure on LTC compared to the rest of the countries. In some Eastern and Southern European countries, such as Cyprus, Bulgaria, Croatia, Greece and Slovakia, the public LTC expenditure is significantly lower than in the above-mentioned countries.

The LTC could be provided by public as well as at private institutions. Regarding the number of formal LTC caregivers, France, Spain and the Netherlands, respectively, show the highest number of personal carers and nurses compared to other countries. Nonetheless, this indicator strongly depends on the population of a country, and in general, countries with smaller populations have less formal LTC carers. Furthermore, data on this subject are missing in some countries, as they have not established records on the number of personal carers and nurses.

Another indicator included in our study is the number of LTC beds in hospitals, which is highest in France, Spain, and in Romania, respectively. Iceland, Malta and Denmark are the countries with the lowest number of LTC beds in hospitals. The indicators included in our study show that the number of LTC beds in nursing and other residential facilities, is highest in countries with the highest population size. Germany, France, the UK, Spain and Italy have the highest number of LTC institutional beds among all countries, while Greece, Malta and Luxembourg have the lowest number of such beds.

Table II presents additional indicators related to the provision of formal LTC that we found in the review (no distinction was made between health care and social care):

- Provision of residential care (at institutions for older persons and nursing homes), home care (health care and social care), day care (semi-residential).

- Coverage rate of residential care and coverage rate of home care (indexes calculated for the population group aged $65+$, with value 1 if equal use by men and women, greater than 1 if higher use by women, and lower than 1 if higher use by men).

- Number of people receiving care in an institution per year. 


\begin{tabular}{|c|c|c|c|c|c|c|c|c|c|c|c|c|c|c|c|c|c|}
\hline 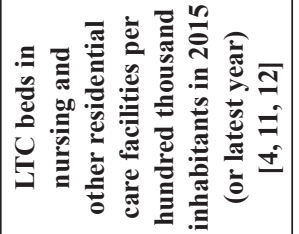 & 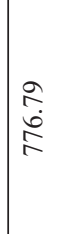 & 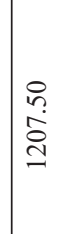 & $\underset{f}{\stackrel{f}{\gamma}}$ & 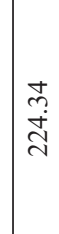 & 导 & \begin{tabular}{l}
$q$ \\
\multirow{2}{*}{} \\
$\tilde{\sigma}$
\end{tabular} & $\begin{array}{l}\hat{0} \\
\dot{0} \\
\infty\end{array}$ & 苛 & $\begin{array}{l}0 \\
2 \\
n \\
n \\
n \\
=\end{array}$ & 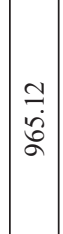 & $\begin{array}{l}\hat{\lambda} \\
\stackrel{\partial}{g}\end{array}$ & 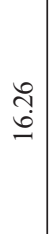 & 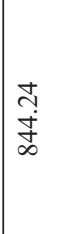 & 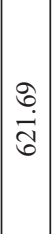 & $\mid \begin{array}{c}0 \\
\infty \\
\infty \\
\infty \\
n\end{array}$ & & 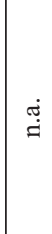 \\
\hline
\end{tabular}

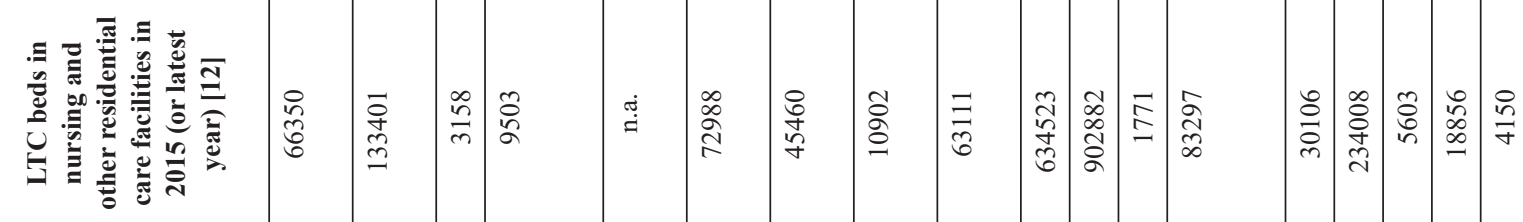

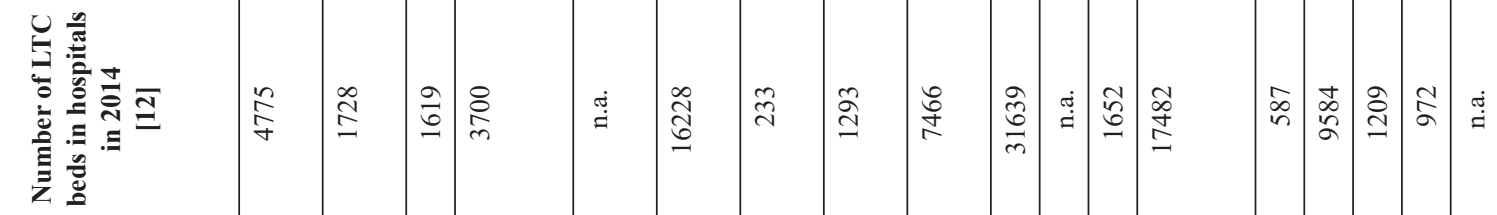

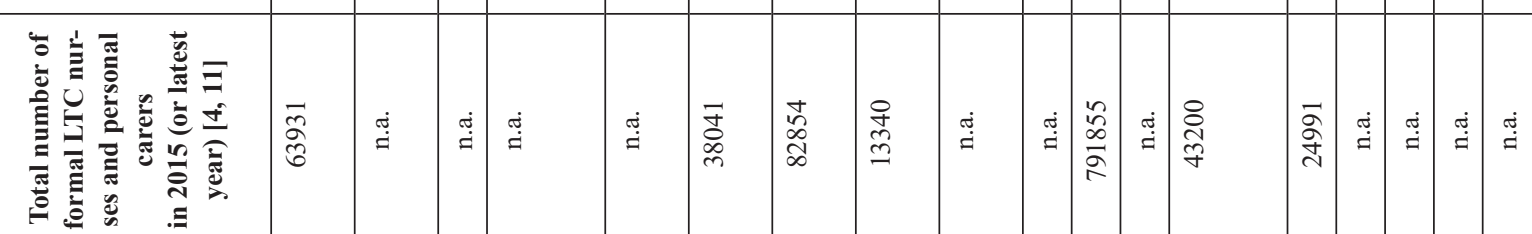

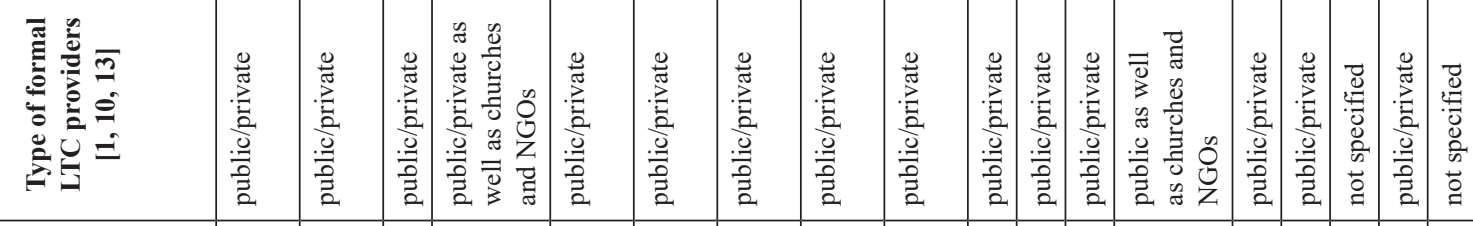

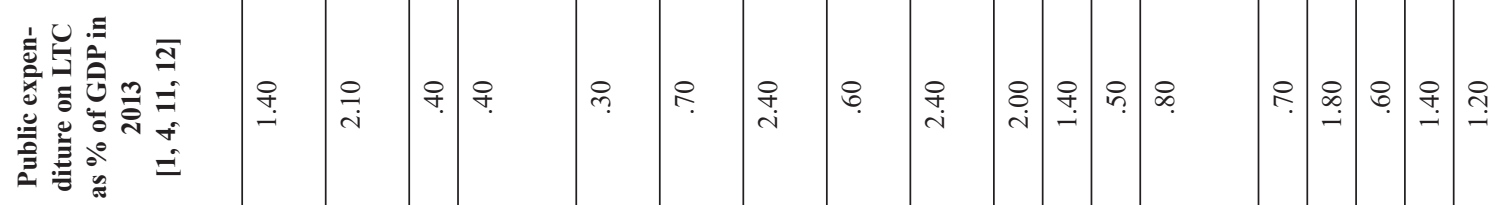

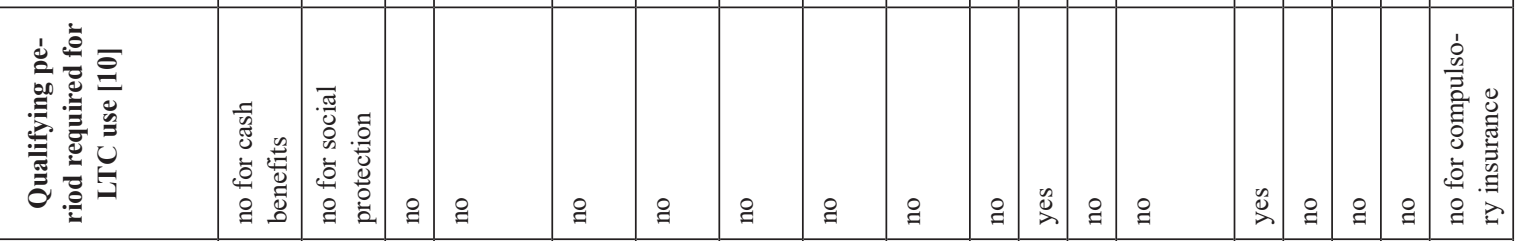

\begin{tabular}{|c|}
\hline 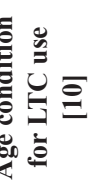 \\
\hline
\end{tabular}

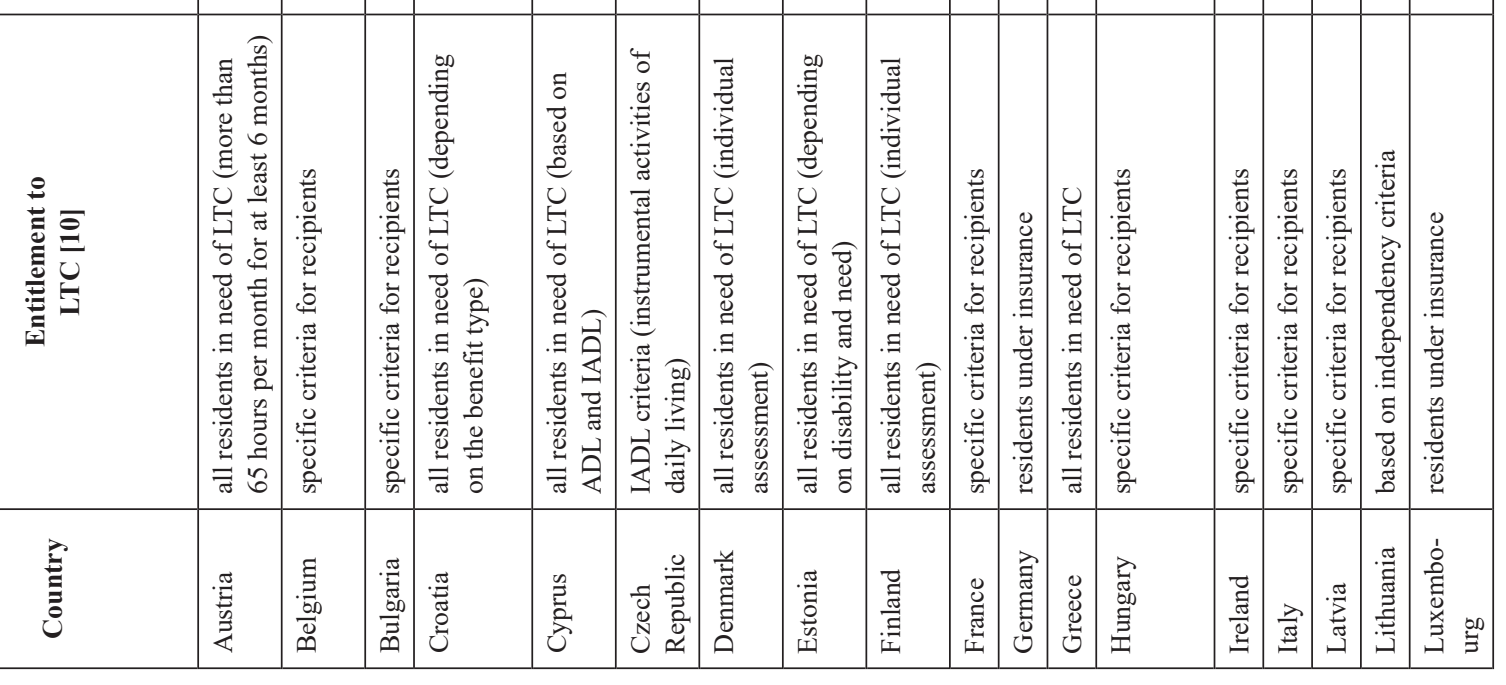




\begin{tabular}{|c|c|c|c|c|c|c|c|c|c|c|c|c|c|}
\hline 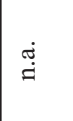 & 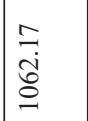 & $\begin{array}{l}\infty \\
\infty \\
\dot{+} \\
\infty \\
-\infty\end{array}$ & $\stackrel{\pi}{=}$ & $\begin{array}{l}\infty \\
\stackrel{\infty}{+} \\
\dot{q}\end{array}$ & $\begin{array}{l}8 \\
i \\
\infty \\
\infty \\
i\end{array}$ & 异 & $\begin{array}{l}m \\
2 \\
\alpha \\
\alpha \\
2\end{array}$ & 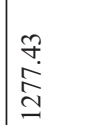 & $\stackrel{\stackrel{\leftrightarrow}{\sharp}}{=}$ & $\stackrel{\stackrel{\leftrightarrow}{\sharp}}{=}$ & \begin{tabular}{|l}
$\vec{m}$ \\
$\infty$ \\
$\stackrel{0}{=}$
\end{tabular} & 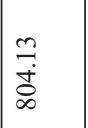 & \\
\hline 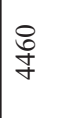 & 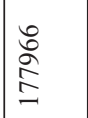 & 송 & $\stackrel{\overbrace{}}{=}$ & 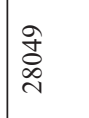 & 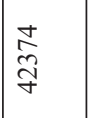 & $\stackrel{\stackrel{\pi}{\sharp}}{=}$ & 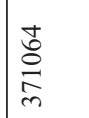 & 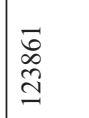 & $\begin{array}{l}\text { के } \\
\text { के } \\
\text { के } \\
\text { in }\end{array}$ & 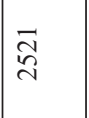 & 客 & $\frac{\rho}{m}$ & \\
\hline ڤ̊ & 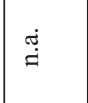 & 胥 & $\stackrel{\pi}{ت}$ & $\overrightarrow{\vec{n}}$ & ¿̊̀ & 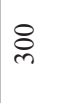 & 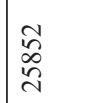 & $\stackrel{\infty}{\bumpeq}$ & $\stackrel{\stackrel{\pi}{\sharp}}{=}$ & $\hat{\mathrm{g}}$ & 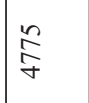 & $\stackrel{\infty}{\stackrel{N}{I}}$ & \\
\hline 㟧 & 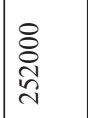 & 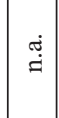 & 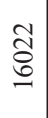 & $\stackrel{\text { g }}{=}$ & 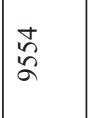 & $\stackrel{\stackrel{g}{\sharp}}{ }$ & $\begin{array}{l}\tilde{n} \\
\tilde{n} \\
\tilde{n} \\
\tilde{m}\end{array}$ & 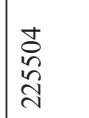 & $\stackrel{\overbrace{}}{g}$ & $\stackrel{\leftrightarrow}{=}$ & 詹 & $\begin{array}{l}8 \\
0 \\
0 \\
0 \\
0\end{array}$ & \\
\hline 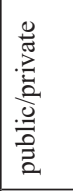 & 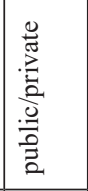 & 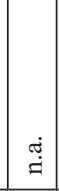 & 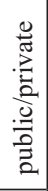 & 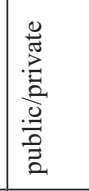 & 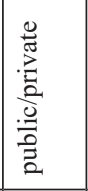 & 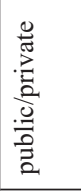 & 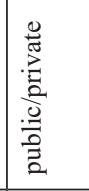 & 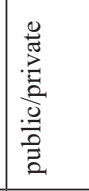 & 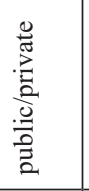 & 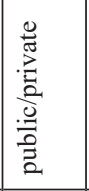 & 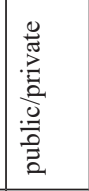 & 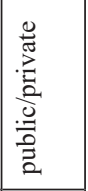 & \\
\hline$\stackrel{\varrho}{\rightleftarrows}$ & $\underset{\text { ঙे }}{\stackrel{+}{+}}$ & 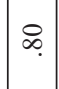 & in & $?$ & ণิ & $\stackrel{\text { q }}{-}$ & $\stackrel{8}{\stackrel{-}{*}}$ & $\underset{\dot{m}}{\stackrel{B}{0}}$ & $\stackrel{\text { ঙิ }}{-}$ & $\stackrel{\infty}{\stackrel{\infty}{\circ}}$ & 忿 & $\hat{n}$ & \\
\hline ‡ & 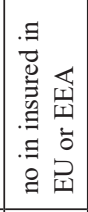 & ‡ & ๑ & 足 & \& & 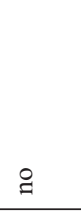 & 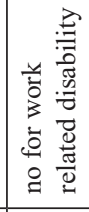 & \& & $\stackrel{\infty}{2}$ & $\stackrel{\infty}{2}$ & 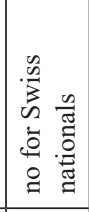 & ‡ & 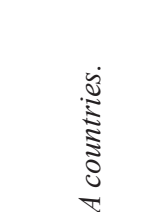 \\
\hline$\stackrel{\infty}{2}$ & 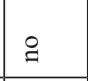 & $\stackrel{\mathscr{D}}{2}$ & ঃ & $\stackrel{\mathscr{D}}{2}$ & $\stackrel{\mathscr{D}}{\curvearrowright}$ & 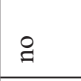 & $\stackrel{\mathscr{D}}{2}$ & 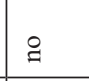 & 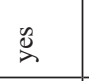 & $\stackrel{9}{9}$ & 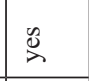 & $\stackrel{9}{9}$ & \\
\hline 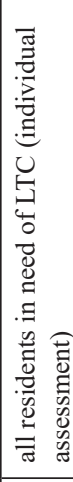 & 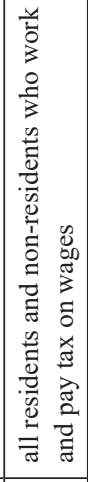 & 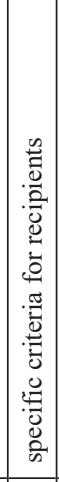 & 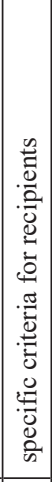 & 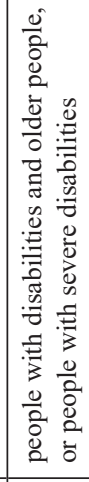 & 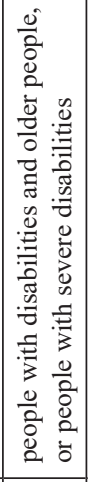 & 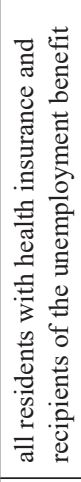 & 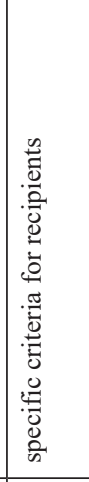 & 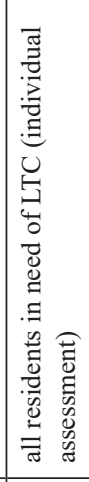 & 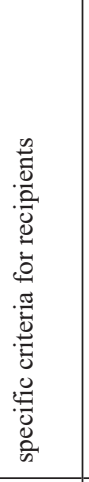 & 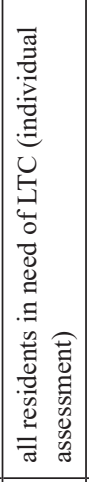 & 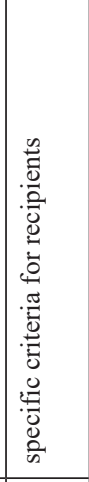 & 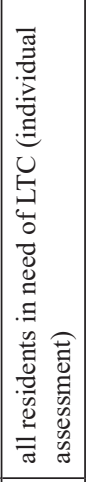 & 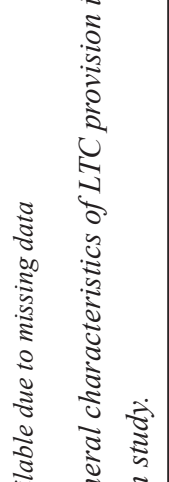 \\
\hline$\frac{\mathbb{F}}{\sigma}$ & 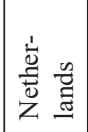 & 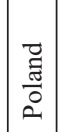 & $\begin{array}{c}\overrightarrow{g_{0}} \\
\stackrel{2}{0} \\
0 \\
0\end{array}$ & 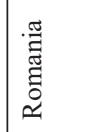 & 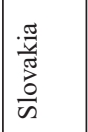 & \begin{tabular}{|l}
.$\frac{\pi}{3}$ \\
$\frac{0}{0}$ \\
$\frac{0}{\omega}$
\end{tabular} & $\begin{array}{l}\text { : } \\
\text { कू. }\end{array}$ & 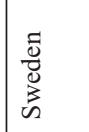 & 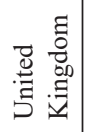 &  & 离 & 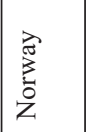 & 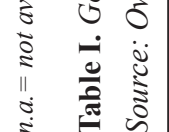 \\
\hline
\end{tabular}




\begin{tabular}{|c|c|c|c|c|c|c|c|c|}
\hline 咅 & 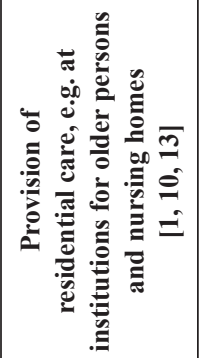 & 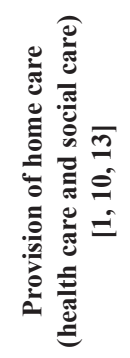 & 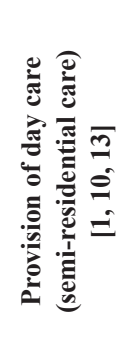 & 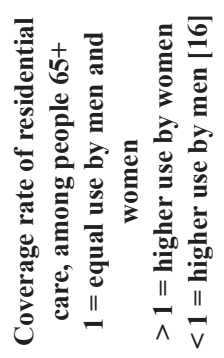 & 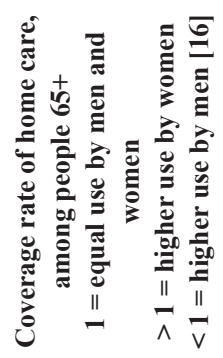 & 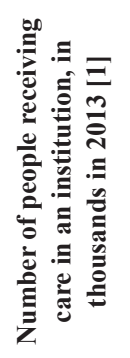 & 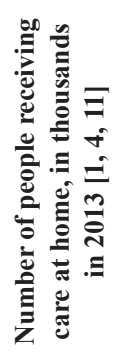 & 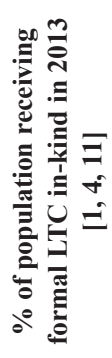 \\
\hline Austria & yes & yes & yes & 3.30 & 14.40 & 74 & 166 & 2.8 \\
\hline Belgium & yes & yes & yes & 6.60 & 7.40 & 143 & 728 & 7.8 \\
\hline Bulgaria & yes & yes & n.a. & n.a. & n.a. & 15 & 106 & 1.7 \\
\hline Croatia & yes & yes & yes & 1.60 & n.a. & 16 & 17 & .8 \\
\hline Cyprus & yes & yes & yes & 3.00 & n.a. & 3 & 3 & .7 \\
\hline $\begin{array}{l}\text { Czech } \\
\text { Republic }\end{array}$ & yes & yes & n.a. & 3.50 & 7.20 & 345 & 94 & 4.2 \\
\hline Denmark & yes & yes & yes & 2.50 & 20.00 & 44 & 101 & 2.6 \\
\hline Estonia & yes & yes & yes & 1.80 & 2.30 & 15 & 6 & 1.6 \\
\hline Finland & yes & yes & yes & 3.10 & 6.30 & 51 & 159 & 3.9 \\
\hline France & yes & yes & yes & 6.70 & 6.50 & 854 & 1089 & 3.0 \\
\hline Germany & yes & yes & yes & 3.50 & 6.60 & 740 & 348 & 1.3 \\
\hline Greece & yes & yes & yes & .60 & 5.60 & 4 & 10 & .1 \\
\hline Hungary & yes & yes & yes & 2.80 & 6.40 & 95 & 61 & 1.6 \\
\hline Ireland & yes & yes & n.a. & 3.90 & 6.50 & 27 & 65 & 2.0 \\
\hline Italy & yes & yes & n.a. & 3.00 & 4.90 & 294 & 754 & 1.8 \\
\hline Latvia & yes & yes & yes & .80 & 1.60 & 11 & 9 & 1.0 \\
\hline Lithuania & yes & yes & n.a. & n.a. & .60 & 61 & 67 & 4.3 \\
\hline $\begin{array}{l}\text { Luxembo- } \\
\text { urg }\end{array}$ & yes & yes & yes & 4.80 & 7.00 & 4 & 9 & 2.4 \\
\hline Malta & yes & yes & yes & 4.30 & 4.00 & 1 & 8 & 2.2 \\
\hline $\begin{array}{l}\text { Nether- } \\
\text { lands }\end{array}$ & yes & yes & n.a. & 6.30 & 21.00 & 383 & 544 & 5.5 \\
\hline Poland & yes & yes & yes & 1.00 & 1.70 & 86 & 118 & .5 \\
\hline Portugal & yes & yes & yes & 3.40 & 4.30 & 23 & 14 & .4 \\
\hline Romania & yes & yes & yes & .50 & .30 & 189 & 204 & 2.0 \\
\hline Slovakia & yes & yes & yes & 3.30 & 2.30 & 45 & 62 & 2.0 \\
\hline Slovenia & yes & yes & yes & 4.80 & 1.80 & 22 & 38 & 2.9 \\
\hline Spain & yes & yes & yes & 4.40 & 4.70 & 307 & 693 & 2.1 \\
\hline Sweden & yes & yes & yes & 5.80 & 9.40 & 87 & 206 & 3.1 \\
\hline $\begin{array}{l}\text { United } \\
\text { Kingdom }\end{array}$ & yes & yes & yes & 4.20 & 6.90 & 243 & 1020 & 2.0 \\
\hline Iceland & yes & yes & yes & 8.30 & 20.50 & 2 & n.a. & .7 \\
\hline $\begin{array}{l}\text { Switzer- } \\
\text { land }\end{array}$ & yes & yes & n.a. & n.a. & n.a. & 90 & 203 & 1.1 \\
\hline Norway & yes & yes & yes & 5.30 & 19.30 & 43 & 94 & .8 \\
\hline
\end{tabular}

n.a. $=$ not available due to missing data

Table II. Provision of formal LTC in the EU and EEA countries.

Source: Own study.

- Number of people receiving care at home per year.

- $\%$ of population receiving formal LTC in-kind.

The table shows that both care at institutions and home care are available in all countries although the ex- act forms of care provision and degree of care availability substantially differ across the countries. The presence of day care is also rather common. However, seven countries have not specified whether they offer this type of 
care. Among the population group aged $65+$, residential care (care at institutions) and home care (care at person's home) are more often used by women than by men, except in Greece, Latvia and Romania in case of residential care, and in Lithuania and Romania in case of home care. The number of people, who receive care in an institution and at home, vary considerably among the countries. Importantly, the share of the population receiving formal LTC in-kind is especially high in Belgium, the Netherlands, Finland and Sweden, as well as in the Czech Republic and Lithuania. This share is much lower in Greece, Portugal, and Poland.

Table III presents indicators of the provision of informal LTC that we found in the review:

- Main providers of informal care.

- Significance of informal care.

- Availability of paid leave option for informal carers.

- Availability of cash or other benefits for informal carers.

- Possibility of flexible work time for informal carers.

- Availability of cash benefits for informal care recipient. The results presented in the table indicate that in all EU Member States, as well as in Iceland, Switzerland and Norway, informal care is provided by family, relatives and by friends. Among the 31 countries, in seven of them, LTC is delivered most commonly by spouses, including the UK, Poland, Norway, Iceland, Switzerland, Greece and Denmark. Bulgaria is the only country that reports informal LTC delivery by individuals taking part in different national social services programs and by unemployed people as personal assistants. Another exception is Croatia, where informal carers are trained by medical doctors to provide the needed care aid.

As indicated in Table III, the indicators included in our study show that informal care has an extremely high role in Italy, and a very essential role in the overall LTC provision in Spain, Finland, Austria, Latvia, Poland, Romania and Slovakia. The rest of the Eastern European countries also indicate a high level of importance of informal care. Almost half of the countries, however, provide no clear indications of the role of informal care due to the lack of information on the number of informal carers. About a third of the countries provide information for temporary paid leave schemes for informal carers, and nearly all countries offer flexible work time as well as some cash or other benefits to the informal carers. However, there is a considerable variation among the countries with regard to the exact benefits and the conditions under which they are offered. About two thirds of the countries offer some cash benefits to the informal care recipient.

Table IV outlines the regulation of quality assurance of formal LTC and the presence of health protection and rehabilitation policies in the EU/EEA area. Among all countries, more than half have quality assurance regulations for residential care. Quality assurance regulations for social home care are present in fifteen countries, including Northern and Western European ones. For nursing home care, more than half of the countries have established quality assurance regulations with the exception of France. However, in most of the Southern and
Eastern European countries, information about the quality assurance regulations is not available.

The data in Table IV also indicate that more than half of the countries have developed health protection and rehabilitation policies related to LTC. Only Italy, Greece, Latvia, and Lithuania lack health protection policies, however, ten countries have provided no information whether they have adopted such policies on LTC. Similarly, half of the countries have not implemented rehabilitation policies related to LTC. Five countries including Belgium, Romania, Slovenia, Spain and Sweden do not have rehabilitation policies in place.

\section{Discussion}

This study has focused on the LTC provision in the EU/ EEA area with an emphasis on differences between the countries in terms of capacity, providers and entitlements. As outlined in the introduction, the motivation behind this study is the growing demand for LTC services as a result of the population ageing [1]. The main study findings are discussed below.

\section{The LIC provision and coverage vary significantly among countries}

Our results suggest that the provision of LTC in Europe involves both public and private providers. The presence of private institutions depicts that the interest in LTC services is common. Private institutions might contribute to higher quality of service provision as long as there are adequate quality monitoring mechanisms in the country's LTC system. As indicated in previous studies, geographic differences in LTC coverage arise especially among private providers, and this may also reflect the differences between rural and urban areas. These differences show that the demand for LTC services is higher in some regions and tends to be lower in others [18].

The use of LTC depends to a large extent on specific eligibility criteria. According to previous analyses, the eligibility criteria for services is often determined at the local level [18]. Our review suggests that most of the countries provide access for all residents in need of LTC. Nevertheless, various definitions of care needs are applied across the countries. Some countries have established specific criteria for using LTC. Overall, the eligibility criteria are underutilized in the LTC systems in the EU and EEA countries.

The country, which spends most on LTC as percentage of GDP, is the Netherlands, followed by other Northern and Western European countries (Sweden, Norway, Switzerland, Denmark and Belgium) that also have high public expenditure on LTC. Overall, Northern and Western European countries generally spend more on LTC from public sources because they invest more in their health care and social care systems. Southern European countries spend considerably less on formal LTC because they strongly rely on the family-based approach to informal care. In some Eastern European countries, public 


\begin{tabular}{|c|c|c|c|c|c|c|}
\hline Country & $\begin{array}{c}\text { Providers of informal care } \\
{[1,10,13]}\end{array}$ & $\begin{array}{c}\text { Role of infor- } \\
\text { mal care } \\
{[1,13]}\end{array}$ & $\begin{array}{c}\text { Paid leave } \\
\text { option for in- } \\
\text { formal carers } \\
{[16]} \\
\end{array}$ & 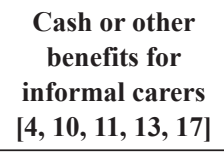 & $\begin{array}{c}\text { Flexible work } \\
\text { time for infor- } \\
\text { mal carers } \\
{[16]} \\
\end{array}$ & $\begin{array}{c}\text { Cash benefits for } \\
\text { informal care } \\
\text { recipient } \\
{[4,10,11,13,17]} \\
\end{array}$ \\
\hline Austria & $\begin{array}{l}\text { family, relatives, friends, } \\
\text { neighbours, volunteers }\end{array}$ & very high & no & yes & yes & yes \\
\hline Belgium & family, relatives, friends, etc. & medium high & no & yes & yes & yes \\
\hline Bulgaria & $\begin{array}{l}\text { family, individuals in diffe- } \\
\text { rent national social services } \\
\text { programs, unemployed } \\
\text { people as personal assistants }\end{array}$ & high & yes & yes & yes & yes \\
\hline Croatia & $\begin{array}{l}\text { family and relatives trained } \\
\text { by doctors }\end{array}$ & n.a. & no & yes & yes & yes \\
\hline Cyprus & family, relatives, friends, etc. & n.a. & no & no & yes & yes \\
\hline $\begin{array}{l}\text { Czech } \\
\text { Republic }\end{array}$ & family, relatives, friends, etc. & n.a. & yes & yes & yes & yes \\
\hline Denmark & $\begin{array}{l}\text { spouses, family, relatives, } \\
\text { friends }\end{array}$ & low & yes & yes & yes & no \\
\hline Estonia & family, relatives, friends, etc. & n.a. & yes & yes & yes & no \\
\hline Finland & family, relatives, friends, etc. & very high & yes & yes & yes & yes \\
\hline France & family, relatives, friends, etc. & n.a. & yes & yes & yes & no \\
\hline Germany & family, relatives, friends, etc. & high & no & yes & yes & yes \\
\hline Greece & $\begin{array}{l}\text { spouses, family, relatives, } \\
\text { friends }\end{array}$ & high & no & no & yes & no \\
\hline Hungary & family, relatives, friends, etc. & high & yes & yes & yes & no \\
\hline Ireland & family, relatives, friends, etc. & n.a. & no & yes & yes & no \\
\hline Italy & family, relatives, friends, etc. & extremely high & yes & yes & yes & yes \\
\hline Latvia & family, relatives, friends, etc. & very high & n.a. & yes & n.a. & no \\
\hline Lithuania & $\begin{array}{l}\text { family, relatives, friends, } \\
\text { neighbours, volunteers }\end{array}$ & n.a. & n.a. & yes & n.a. & yes \\
\hline Luxembourg & family, relatives, friends, etc. & n.a. & n.a. & yes & n.a. & yes \\
\hline Malta & family, relatives, friends, etc. & high role & no & yes & yes & no \\
\hline Netherlands & family, relatives, friends, etc. & n.a. & yes & no & yes & yes \\
\hline Poland & $\begin{array}{l}\text { spouses, family, relatives, } \\
\text { friends }\end{array}$ & very high & yes & yes & yes & yes \\
\hline Portugal & $\begin{array}{l}\text { family, relatives, friends, } \\
\text { neighbours, volunteers }\end{array}$ & n.a. & yes & no & yes & yes \\
\hline Romania & family, relatives, friends, etc. & very high & yes & yes & yes & yes \\
\hline Slovakia & family, relatives, friends, etc. & very high & no & yes & yes & yes \\
\hline Slovenia & family, relatives, friends, etc. & n.a. & yes & yes & yes & yes \\
\hline Spain & family, relatives, friends, etc. & very high & no & yes & yes & yes \\
\hline Sweden & family, relatives, friends, etc. & n.a. & yes & yes & yes & yes \\
\hline $\begin{array}{l}\text { United } \\
\text { Kingdom }\end{array}$ & $\begin{array}{l}\text { spouses, family, relatives, } \\
\text { friends }\end{array}$ & n.a. & n.a. & yes & n.a. & yes \\
\hline Iceland & $\begin{array}{l}\text { spouses, family, relatives, } \\
\text { friends }\end{array}$ & high & no & yes & yes & no \\
\hline Switzerland & $\begin{array}{l}\text { spouses, family, relatives, } \\
\text { friends }\end{array}$ & high & n.a. & yes & n.a. & yes \\
\hline Norway & $\begin{array}{l}\text { spouses, family, relatives, } \\
\text { friends }\end{array}$ & n.a. & no & yes & yes & yes \\
\hline
\end{tabular}

n.a. = not available due to missing data

Table III. Provision of informal LTC in the EU and EEA countries.

Source: Own study. 


\begin{tabular}{|c|c|c|c|c|c|}
\hline Country & $\begin{array}{c}\text { Regulation of quality } \\
\text { assurance related to } \\
\text { residential care } \\
{[1,14,15]}\end{array}$ & $\begin{array}{c}\text { Regulation of quality } \\
\text { assurance related to } \\
\text { social home care } \\
{[1,14,15]}\end{array}$ & $\begin{array}{c}\text { Regulation of quality } \\
\text { assurance a related } \\
\text { to nursing home care } \\
{[1,14,15]}\end{array}$ & $\begin{array}{c}\text { Health protection } \\
\text { policy } \\
{[1,13]}\end{array}$ & $\begin{array}{c}\text { Rehabilitation policy } \\
{[11,13]}\end{array}$ \\
\hline Austria & yes & yes & yes & n.a. & n.a. \\
\hline Belgium & yes & yes & yes & yes & no \\
\hline Bulgaria & n.a. & n.a. & n.a. & yes & yes \\
\hline Croatia & n.a. & n.a. & n.a. & yes & yes \\
\hline Cyprus & n.a. & n.a. & n.a. & yes & yes \\
\hline Czech Republic & partly & yes & yes & n.a. & n.a. \\
\hline Denmark & yes & yes & yes & yes & yes \\
\hline Estonia & n.a. & n.a. & n.a. & n.a. & n.a. \\
\hline Finland & n.a. & n.a. & n.a. & yes & yes \\
\hline France & yes & no & no & yes & yes \\
\hline Germany & yes & yes & yes & n.a. & n.a. \\
\hline Greece & n.a. & n.a. & n.a. & no & yes \\
\hline Hungary & yes & no & yes & yes & yes \\
\hline Ireland & n.a. & n.a. & n.a. & yes & yes \\
\hline Italy & n.a. & n.a. & n.a. & no & yes \\
\hline Latvia & yes & yes & yes & no & yes \\
\hline Lithuania & yes & yes & yes & no & yes \\
\hline Luxembourg & n.a. & n.a. & n.a. & n.a. & n.a. \\
\hline Malta & n.a. & n.a. & n.a. & yes & yes \\
\hline Netherlands & yes & yes & yes & n.a. & n.a. \\
\hline Poland & n.a. & n.a. & n.a. & n.a. & n.a. \\
\hline Portugal & n.a. & n.a. & n.a. & yes & yes \\
\hline Romania & yes & no & yes & yes & no \\
\hline Slovakia & yes & yes & yes & yes & yes \\
\hline Slovenia & yes & yes & yes & yes & no \\
\hline Spain & yes & yes & yes & yes & no \\
\hline Sweden & yes & yes & yes & yes & no \\
\hline UK & yes & no & yes & yes & yes \\
\hline Iceland & yes & yes & yes & n.a. & n.a. \\
\hline Switzerland & yes & yes & yes & n.a. & n.a. \\
\hline Norway & yes & yes & yes & n.a. & n.a. \\
\hline
\end{tabular}

n.a. $=$ not available due to missing data

Table IV. Regulation of quality assurance of formal LTC, health protection and rehabilitation policies in the EU and EEA countries. Source: Own study.

expenditure on LTC is also relatively low. Although the approach to LTC provision depends on cultural factors and historical development, countries' fiscal capacities also play an important role. The fiscal efficiency of the LTC systems could be an important indicator for policymakers, especially in countries, which need to generate additional resources for LTC provision.

The number of personal carers and nurses delivering LTC is related to the population size of a country. Thus, countries with a higher population size typically have a higher number of LTC personal carers and nurses, though this is not always applicable since the Netherlands with medium population size, stands out as a country with one of the highest number of caregivers. However, we lack information for seventeen countries regarding this indicator and hence, this prevents any generalisations about the relation between the population size and the number of LTC carers.

Despite the great diversity in the characteristics of the LTC systems, it is possible to distinguish countries that are quite liberal on the access to LTC and spend a high share of GDP on LTC services, as well as countries that have very specific criteria for access to LTC services and low LTC spending. There are also several countries that fall between these two extremes. 


\section{The provision of formal LIC is especially high in Western European countries and mostly low in Eastern European countries}

Residential care and home care are available in all countries. Day care is very common. In general, well-funded Western European countries have higher use of residential care. The use of formal LTC is especially high in Belgium, the Netherlands, Sweden and Finland. These countries have well-organised health care and social care sectors, which are able to support the provision of LTC at the homes of users or in institutions. However, the use of formal LTC is lower in Southern and Eastern European countries (e.g. Greece, Portugal and Poland). These countries suffer underfunding and lack resources within their health care and social care systems. This indicates the need for governments to secure additional LTC resources and adopt policy measures to enhance the LTC service provision.

Southern and Eastern European countries have the lowest rate of formal care according to this study. The reasons for the low coverage may be explained by the lack of care personnel or by high costs of employing a care giver. LTC users in Eastern Europe usually receive low pensions, and in most cases, it is impossible to pay on their own for formal home care, unless they receive financial support from their family [19].

The results on the provision of formal LTC indicate that a majority of Eastern European countries need to consider ways to improve the sustainability and efficiency of their LTC systems and respond to the challenges of formal LTC. This could include the establishment of new residential homes, employment of well-trained personal caregivers and nurses qualified in LTC, providing in-kind benefits, as well as health protection and rehabilitation services. This will however require substantial LTC investments on a long term.

\section{The provision of informal care is essential in Southern and Eastern European countries, but it also plays an important role in some Western European countries}

Despite the general understanding that formal LTC provides good quality and accessible services among recipients, many Europeans rely on informal care. Since formal LTC requires financial support and government resources, when those support and resources are absent, persons who need LTC are often unable to pay for such services. The lack of money to pay for formal care limits the provision of formal care. The inability to pay for formal LTC also increases the use of informal care across many EU countries [1]. Hence, LTC is traditionally associated with informal care provision in these countries.

The results of this study confirm that informal care is provided by family, relatives and by friends in all examined countries. Moreover, the findings indicate that the role of informal care in the EU/EEA area is increasing regardless the geographical location. Informal care is frequent and highly important in Southern and Eastern European countries (e.g. Bulgaria, Greece, Hungary,
Poland), but also in Northern and Western European countries (e.g. Finland, Germany, Switzerland, Ireland, and Iceland). Almost half of the countries however, have no data on the role of informal care and there is a lack of information on the number of informal carers. Further assessment of the provision of informal care could facilitate effective strategies and policy measures to improve the quality and effectiveness of this type of care.

As indicated in the literature, mechanisms for supporting informal carers such as cash-benefits are an important factor, which can significantly reduce the LTC costs and stimulate the use of informal care if informal carers can be compensated [18]. In majority of the analysed countries, there are cash benefits available for cate givers and flexible work arrangements. In addition, almost half of the countries provide a paid leave option for informal carers from their jobs. Although benefits for informal carers are becoming common, policy makers in Eastern European countries are challenged to adopt effective measures to adequately compensate the use of informal care.

Considering these circumstances, it could be highlighted that the notions of family responsibility are clearly related to the LTC expenditure. In countries where informal care is more common, there is a need to improve the financing of formal care provision and to enhance the access to public care services, despite the key role of informal care [19]. Research on the provision of informal care is based on indicators, which might have limited accuracy due to the subjectivity of measuring the role in informal care. Thus, along with the recipients and providers of LTC, studies on the role in informal care could also target other stakeholders, policy-makers, health insurers, and representatives of non-governmental organisations (NGO).

\section{Most of the countries have implemented LIC quality assurance regullations}

The last part of the results examined the regulations of quality assurance of formal LTC, and presence of health protection and rehabilitation policies. We find that quality assurance regulations for home care are common in Northern and Western European countries. Although many countries in the EU/EEA area have quality assurance regulations for residential LTC care, most of the Eastern European and Southern European countries lack information about the quality assurance regulations. Eastern European countries generally have less LTC-related regulations in comparison with countries in the rest of Europe. As a result, there is a lack of monitoring and control mechanisms on the LTC systems in Eastern European countries.

\section{The division between social support services and health-related services is essential}

When analysing the provision of LTC, it is essential to distinguish between the provision of social support services and health-related services. Recipients of LTC 
could make the choice between the types of care according to their needs and levels of disability to perform daily activities. Too often, data on the provision of LTC lack concrete information whether providers are delivering medical (health-related) or non-medical (social support) services. It is known that several countries in the EU/ EEA area focus more on outpatient rehabilitation treatment at an earlier stage of chronic diseases compared to other countries that mainly concentrate on providing LTC in hospitals or clinics [20]. A major drawback of the data used in this study is that they do not specify whether the providers are delivering medical treatment and supervision, or if they are delivering social services, such as help with personal care or cleaning, helping with groceries, cooking. In order to reduce the gap between the provision of medical LTC and social LTC, it is recommended that providers report to the national authorities, the types of care, which they provide. Such data collected at the national level could help to make future cross-country comparisons and country-level assessments more accurate.

\section{Discussion of study design}

This study outlines the provision of both formal and informal LTC and it takes into consideration the link between them. Another advantage is that it combines data on LTC provision from reliable international databases such as the OECD Stats, EUROSTAT, the WHO country reports. The collection of all indicators enables to make general statements about the LTC systems across countries. The study identifies the need of policy discussions in countries where LTC is poorly financed and regulated.

Nonetheless, there are several study limitations and the most crucial one is the scarcity of data and evidence related to the topic. There is a need of formal channels to report LTC-related data on an annual base. Such data could be gathered locally for example by municipalities and then transferred to the government to be reported to international agencies that monitor LTC systems and developments. In addition, country-based surveys could be carried out to measure the provision and quality of LTC services. In order to create trustworthy typologies of LTC provision, it is crucial to have the most recent and accurate data for all counties.

\section{Conclusion}

This study highlighted the provision of formal and informal LTC across the countries in the EU/EEA area using macro-level data. The results and their discussion helped to enhance the knowledge and understanding of the LTC systems in general. However, due to the scarcity of information, further research is needed to explore the provision of formal and informal LTC, as well as the LTC coverage and arrangements. Furthermore, the division of social support services and health-related services is essential to make country comparisons more appropriate and legit. Countries should clarify whether LTC is part of the health care system or the social services system. Next to this, International databases like OECD Stats and
EUROSTAT should facilitate an enhanced monitoring of countries' LTC systems. Precise and relevant LTC data could enable LTC developments through comparative cross-country analyses.

Another indicator that is essential for measuring the provision of LTC, is the mechanism of paying the professional caregivers (e.g. nurses). In this study, it was not possible to find reliable data on provider payment mechanisms across the different countries. This indicator is particularly important in a cross-country analysis to determine optimal payment mechanisms for the providers of care. An effective provider payment mechanism could increase the motivation of personal carers and nurses, which would then lead to an improvement in the quality and efficiency of formal and informal LTC services.

Responding to the challenges of the LTC systems requires comprehensive, effective and well-coordinated actions and reforms in all EU/EEA countries, especially in Eastern and Southern Europe. Ensuring more age-friendly and disability-friendly, as well as barrier-free environments, could enhance LTC users to live independently. Health protection and rehabilitation services are another essential measure to enable LTC users to maintain good health and well-being. Policy-makers should particularly focus on health protection and rehabilitation strategies and programs in every EU/EEA country to increase the quality of life and wellbeing of older citizens [19]. To assure comparable data on LTC provision across EU/EEA countries, a plausible option is to develop and implement an e-LTC platform to enter, save and store information on LTC provision.

\section{References}

1. European Commission, Joint Report on Health Care and Long-Term Care Systems \& Fiscal Sustainability. Volume 1 and Volume II. INSTITUTIONAL PAPER 037, 2016, https://ec.europa.eu/info/publications/economy-finance/ joint-report-health-care-and-long-term-care-systems-fiscalsustainability-0_en (accessed: 08.01.2017).

2. European Commission, Population Ageing in Europe: Facts, Implication and Policies. Outcomes of EU-funded research, Directorate-General for Research and Innovation, 2014, https://webcache.googleusercontent.com/search?q=cache:jW_ v1-A4aQ4J:https://ec.europa.eu/research/social-sciences/pdf/ policy_reviews/kina26426enc.pdf $+\& c d=2 \& h l=n 1 \& c t=$ clnk $\&$ $\mathrm{gl}=\mathrm{n} 1 \& \mathrm{client}=$ firefox-b-ab (accessed: 08.01.2017).

3. WHO Regional Office for Europe, Health and Social Care Systems, http://www.euro.who.int/en/health-topics/Lifestages/healthy-ageing/data-and-statistics/health-and-socialcare-systems (accessed: 22.01.2017).

4. Colombo F., Llena-Nozal A., Mercier J., Tjadens F., Help Wanted? Providing and Paying for Long-Term Care. OECD Health Policy Studies, OECD Publishing, 2011, http:// www.keepeek.com/Digital-Asset-Management/oecd/socialissues-migration-health/help-wanted 9789264097759-en\# page41 (accessed: 22.01.2017).

5. OECD, Long-Term Care for Older People, 2005, https:// eclass.unipi.gr/modules/document/file.php/SAE148/ 


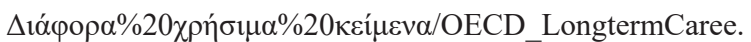
pdf (accessed: 22.01.2017).

6. World Health Organization, WHO Health Systems Framework, http://www.wpro.who.int/health_services/health_systems_framework/en/ (accessed: 22.01.2017).

7. McCall N., Long-Term Care: Definition, Demand, Cost, and Financing, in: N. McCall (ed.), Who Will Pay for Long Term Care? Insights from the Partnership Programs, Health Administration Press, Chicago; Academy for Health Services Research and Health Policy, Washington, DC 2001.

8. Salvador-Carulla L. et al., Evaluation of an integrated system for classification, assessment and comparison of services for long-term care in Europe: The eDESDE-TC study, "BMC Health Services Research" 2013; 13: 218.

9. Kraus M., Riedel M., Mot E., Willeme P., Rohring G., Czypionka T., A Typology of Long-Term Care Systems in Europe, European Network of Economic Policy Research Institutes, 2010, http://www.ancien-longtermcare.eu/ (accessed: 28.01.2017).

10. MISSOC, Comparative Tables, 2016, http://www.missoc. org/MISSOC/INFORMATIONBASE/COMPARATIVETA BLES/MISSOCDATABASE/comparativeTablesSearchResultTree.jsp (accessed: 22.01.2017).

11. OECD, OECD Stats, http://stats.oecd.org/ (accessed: 22.01. 2017).

12. European Commission, EUROSTAT, http://ec.europa.eu/ eurostat (accessed: 22.01.2017).

13. European Observatory on Health Systems and Policies, Health system reviews (HiT series), http://www.euro.who.int/ en/about-us/partners/observatory/publications/health-systemreviews-hits (accessed: 22.01.2017).

14. Dandi R., Casanova G., Quality Assurance Indicators of Long-Term Care in European Countries, European Network of Economic Policy Research Institutes, 2012, http://www. ancien-longtermcare.eu/node/27_(accessed: 28.01.2017).
15. Dandi R., Casanova G., Lillini R., Volpe M., De Belvis A.G., Avolio M., Pelone F., Long-Term Care Quality Assurance Policies in European Countries, ENEPRI Research Report No. 111. Work Package 5. European Network of Economic Policy Research Institutes, 2012, http:/www. ancien-longtermcare.eu/node/27 (accessed: 28.01.2017).

16. European Commission, Long-Term Care for the Elderly. Provision and Providers in 33 European Countries, Publication of the European Union, Luxembourg 2012, https://ec.europa. eu/justice/gender-equality/files/elderly_care_en.pdf (accessed: 25.01.2017).

17. Riedel M., Kraus M., Informal Care Provision in Europe: Regulation and Profile of Providers, ENEPRI Research Report No. 96, European Network of Economic Policy Re-

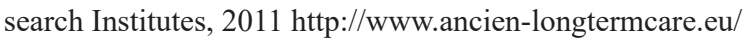
node/27 (accessed: 25.01.2017).

18. European Commission, Analysing Equity in the Use of Long-Term Care in Europe, Research note 9/2014, Directorate-General for Employment, Social Affairs and Inclusion, 2014, www.euro.centre.org/data/1429795073_77691.pdf (accessed: 25.01.2017).

19. Verbeek-Oudijk D., Woittiez I., Eggink E., Putman L., Who Cares in Europe? A Comparison of Long-Term Care for the Over-50s in Sixteen European Countries, The Netherlands Institute for Social Research, 2014, http://webcache.googleusercontent.com/search?q=cache:r7bA4wDZY1QJ:www. scp.nl/dsresource\%3Fobjectid\%3Dd9b6eed0-a19b-4646-8c $\mathrm{d} 2-\mathrm{db} 24 \mathrm{~d} 20 \mathrm{e} 98 \mathrm{~d} 9+\& \mathrm{~cd}=1 \& \mathrm{hl}=$ bg \& ct=clnk \&gl=bg\&client =firefox-b-ab (accessed: 25.01.2017).

20. European Commission, Long-Term Care in the European Union, Office for Official Publication of the European Communities, Luxembourg 2008, http://ec.europa.eu/ social/BlobServlet?docId=2781\&langId=en (accessed: 09.01.2017). 